\title{
An Event-Related fMRI Study of Self-Paced Writing of Simple Dots
}

\author{
I. Rektor ${ }^{1}$, I. Rektorová ${ }^{1}$ M. Mikl ${ }^{1}$, M. Brázdil' ${ }^{1}$, and P. Krupa ${ }^{2}$ \\ ${ }^{1}$ First Department of Neurology and ${ }^{2}$ Diagnostic Imaging Department, \\ Masaryk University, St. Anne's Hospital, Brno, Czech Republic
}

\begin{abstract}
The spatial locations of brain activation related to self-paced repetitive movements, i.e., writing simple dots, were studied using event-related fMRI. Ten healthy right-handed subjects were scanned while performing the protocol with self-paced repetitive movement. In a protocol with self-paced dot writing, we observed significant activation in regions known to participate in motor control: Contralateral to the movement in the primary sensorimotor and supramarginal cortices, the supplementary motor area (SMA) and the underlying cingulate, and, to a lesser extent, the sensorimotor region ipsilateral to the writing hand. When the fMRI was compared with the map of the brain areas electrically active with self-paced movements (intracerebral recordings; Rektor et al., 1994, 1998, 2001, b, c, 2003), there was an evident overlap of most results. Nevertheless, the electrophysiological studies were more sensitive in uncovering small active areas, i.e., in the premotor and prefrontal cortices. The Bereitschafspotential (BP) with Movement Accompanying Potential (MAP) and hemodynamic changes overlap in regions where the BP were displayed consistently. In some other regions, the BP recordings were inconsistent, i.e., in the prefrontal cortex, where about half of the contacts displayed BP generators while the other half did not. In these regions the hemodynamic changes were not significant. The spatial limitations of the intracerebral electrodes is a result of the fact that the electrodes are submerged in the cerebral tissue and record in their immediate vicinity. The fMRI that indirectly measures activity of larger populations of neurons has better spatial resolution. Electrophysiological techniques with intracranial recordings may reveal even subtle generators of electrical activity.
\end{abstract}

Keywords: self-paced, writing dots, fMRI, Bereitschaftspotential, movement accompanying potential

\section{Introduction}

The preparation and execution of self-paced movement has been studied since the discovery of Bereitschafspotential (BP) in 1964 (Kornhuber \& Deecke 1965). In a series of studies with intracerebral recordings of brain electrical activity, we have mapped brain activity related to simple and complex self-paced movements, and our knowledge of the map of electrically active brain areas in this kind of protocol is quite reliable (Rektor et al., 1994, 1998, 2001b, c, 2003).

The introduction of new imaging and metabolic techniques such as PET and fMRI has made it possible to complement the information revealed by electrophysiological measuring. Intracerebral recording alone may not explore the distributed neural system in its whole complexity. Multiple techniques, including those with poorer temporal resolution but with better spatial resolution (fMRI, PET), are required in order to completely understand the roles of various structures in the cognitive processes. On the other hand, the recording of metabolic and hemodynamic changes is hardly sufficiently sensitive to display subtle changes in activity patterns inside individual brain regions. The limited spatial resolution of intracerebral recording may be partially overcome by a larger number of recordings. Direct recording from cerebral structures remains an important and as yet irreplaceable source of information.

A simple self-paced movement was studied with fMRI in several studies (Ball et al., 1999; Maccotta et al., 2001; Newton, 2002; Cunnington et al., 2003). The results of fMRI and BP studies concurred in revealing activation of several areas, but differed in revealing activity in some others. There have been discrepancies between the results of various fMRI studies. Similarly, when the results of electrophysiology and fMRI have been compared using other protocols, some overlap and some discrepancies were described (Brázdil et al., 2005). In this study, we compared the changes in brain regional perfusion (fMRI) with intracerebrally recorded local field potentials in the BP protocol. A simple but meaningful movement - writing dots was chosen for the fMRI study of healthy volunteers, while in the BP studies of epilepsy surgery candidates both simple (flexion of the hand or foot) and meaningful complex movements (turning pages in a book) were tested. We compared various types of movements after we could describe the identical anatomical distribution of BP with simple and with complex movements in a previous depth electrode study (Rektor et al., 2001a). 


\section{Materials and Methods}

\section{Subjects}

Ten volunteers participated in our study, all right-handed, healthy subjects (two females, eight males) from the professional or academic sector, none of whom had a history of neurological or psychiatric disease. The mean age was $23.5 \pm 1.28(S D)$ years, ranged from 20 to 25 years. Informed consent was obtained from each subject after all of the procedures had been fully explained, and the study received the approval of the local ethics committee.

\section{Task}

Subjects were instructed to fix their gaze on the line at the top of the MR gantry, and to keep their eyes open. Since the subject was supine in the scanner, he could not see what he was writing. The overall duration of the functional acquisitions was $6 \mathrm{~min}$. Subjects were instructed to write dots. They were asked to write the dots slowly, holding the pen on the tablet for a while. The subject determined the start of the trial. Subjects were instructed not to perform the requested task more than once in $20 \mathrm{~s}$, to write the dots without breaking the contact of the special opto-mechanical pen on the tablet, and to minimize hand movements in the rest periods. The subjects were asked to estimate the approximate interval covertly, without any external or internal cue. The subjects were asked not to count the time, but to make the requested movement after an approximately estimated period of time had passed.

The tablet was held in the subject's left hand, and the pen was held in the subject's right hand. The tablet rested freely on the subject's abdomen. The opto-mechanical pen was equipped with a touch scanner, and was connected to the computer via fiber optic cable. The computer recorded information about the initialization and completion of contact between the pen and the pad. An interview was performed with each subject after the examination to evaluate the subject's assessment of the task qualities. The mean periods between trials was $28.7 \pm 9.6 \mathrm{~s}$. The mean number of successful trials was $11.4 \pm 3.9$.

\section{MR Acquisition}

Imaging was performed on a $1.5 \mathrm{~T}$ Siemens Magnetom Symphony scanner. Gradient echoplanar (EPI) imaging was used for functional images: TR (scan repeat time) = $2760 \mathrm{~ms}$ (including $1 \mathrm{~s}$ of silence), $\mathrm{TE}=40 \mathrm{~ms}$, flip angle $=$ $90^{\circ}, \mathrm{FOV}=230 \mathrm{~mm}$, slice thickness $=5 \mathrm{~mm}$, interslice gap $=2 \mathrm{~mm}, 16$ transversal slices, inplane resolution $64 \times$ 64. A total of 130 functional scans were acquired from each subject for the overall study. Following functional measurements, high-resolution T1-weighted anatomical imag- es were collected using a 3D sequence with the following parameters: 160 sagittal slices, inplane resolution $512 \times$ 512 , slice thickness $=1.17 \mathrm{~mm}, \mathrm{TR}=1700 \mathrm{~ms}, \mathrm{TE}=$ $3.96 \mathrm{~ms}, \mathrm{FOV}=246 \mathrm{~mm}$, flip angle $15^{\circ}$.

\section{fMRI Data Analysis}

fMRI data were analyzed using the SPM99 (Wellcome Department of Cognitive Neurology, London, UK) program, using a statistical parametric mapping and general linear model. Several scans from each functional series (as many as needed for excluding the first event) were excluded from the analysis. Functional scans were realigned (motion correction), normalized to the Montreal Neurological Institute (MNI) template, and smoothed (using a Gaussian filter with a full width at half maximum of $6 \mathrm{~mm}$ ). Functional series were filtered with a high-pass filter (cut-off period of $90 \mathrm{~s}$ ) and a low-pass filter (Gaussian kernel with a full width at half maximum of $4 \mathrm{~s}$ ). Canonical Heamodynamic Response Function (HRF) and its temporal and dispersion derivatives were used as regressors of interest, and movement parameters were used as regressors for excluding movement-related effects. The tcontrast files were generated individually for each regressor (dot-canonical hrf, dot-temporal derivative, dot-dispersion derivative), for use in second-level analysis. This random effect analysis was made using ANCOVA with the three regressors of interest (canonical HRF, its temporal and dispersion derivatives) and mean trial duration per subject as a covariate for reduction of its impact on group analysis. We used a statistical threshold of $p<.05$ corrected for multiple comparisons.

\section{Results}

In the performed task, significant fMRI activations were found in several brain regions, with obvious left-sided predominance. The clusters of activation with the most significant findings were revealed in the pericentral region, inferior parietal gyrus, and middle cingulate on the left side (Figure 1). Table 1 summarizes the anatomical areas, their MNI coordinates, and statistical scores of the regions with significant activation in the investigated task.

\section{Discussion}

We investigated repetitive movements in a self-paced paradigm. The movement was simple but meaningful: Making a dot on a tablet with an opto-mechanical pen. Selfinitiated, simple repetitive movements have often been used as an electrophysiological tool for investigating movement preparation and execution in the Readiness Po- 


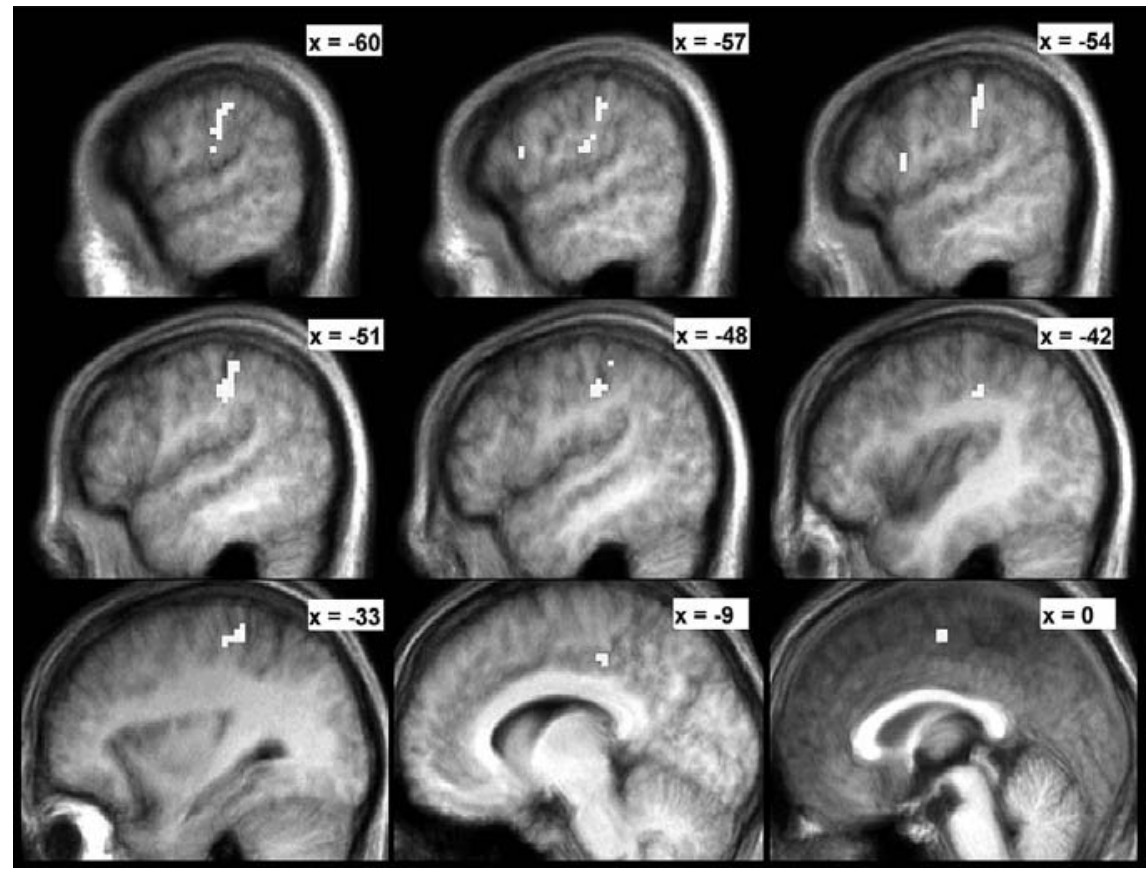

Figure 1. Group activation map for writing dots, overlapped on anatomical MR scans (mean of all volunteers). Significance threshold $p<.05$, corrected.

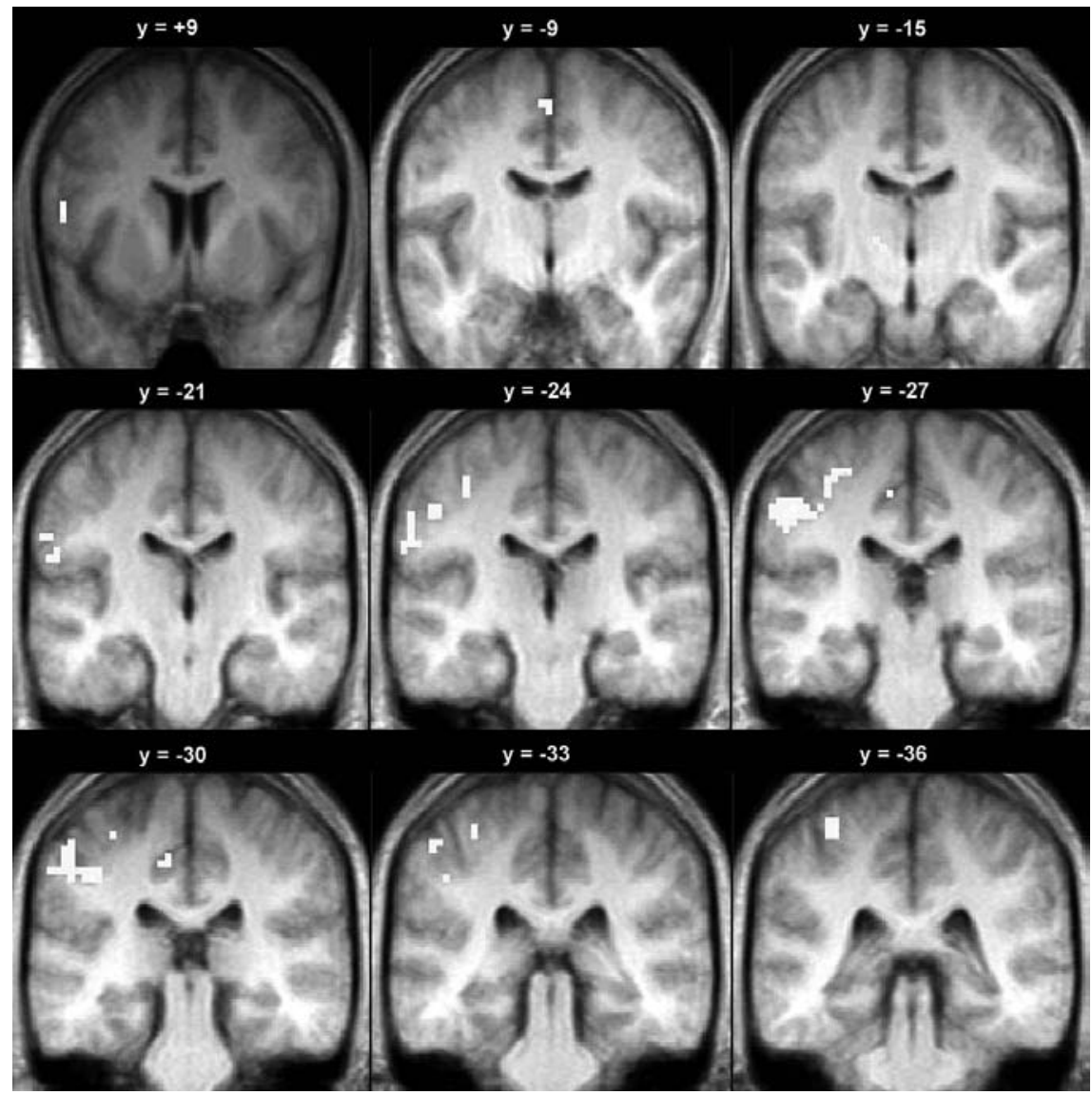

tential (Bereitschaftspotential, BP) protocol. BP also covers activities that could be considered cognitive: The timing, the decision to perform the movement, the preparation for and the initiation of the motion (Rektor et al., 2001a; Libet, 1985). The premovement potential (BP) is typically followed by a complex potential, which is recorded during the movement, and which we have referred to as the Movement Accompanying Potential (MAP; Rek- 
Table 1. Localization of significant activated areas. Significance threshold $p<.05$, corrected

\begin{tabular}{|c|c|c|c|c|c|c|}
\hline \multirow[t]{2}{*}{ Brain region } & \multirow[t]{2}{*}{ BA } & \multicolumn{3}{|c|}{ MNI coordinates } & \multirow[t]{2}{*}{$z$-score } & \multirow[t]{2}{*}{$F$-score } \\
\hline & & $\mathrm{x}$ & $\mathrm{y}$ & $\mathrm{z}$ & & \\
\hline Left Postcentral & 2,1 & -51 & -27 & 42 & 5.52 & 38.59 \\
\hline Left Frontal inf. & 44 & -54 & 9 & 15 & 5.39 & 35.64 \\
\hline Left Parietal inf. & 40 & -42 & -30 & 39 & 5.38 & 35.46 \\
\hline Left Postcentral & $1,2,3$ & -33 & -36 & 60 & 5.36 & 35.03 \\
\hline Left Postcentral & 2 & -60 & -24 & 30 & 5.30 & 33.93 \\
\hline Left Cingulate & 31 & -9 & -30 & 45 & 5.05 & 29.18 \\
\hline Left SMA & 6 & 0 & -9 & 60 & 5.02 & 28.79 \\
\hline Left Precentral & 4 & -33 & -27 & 57 & 4.96 & 27.70 \\
\hline
\end{tabular}
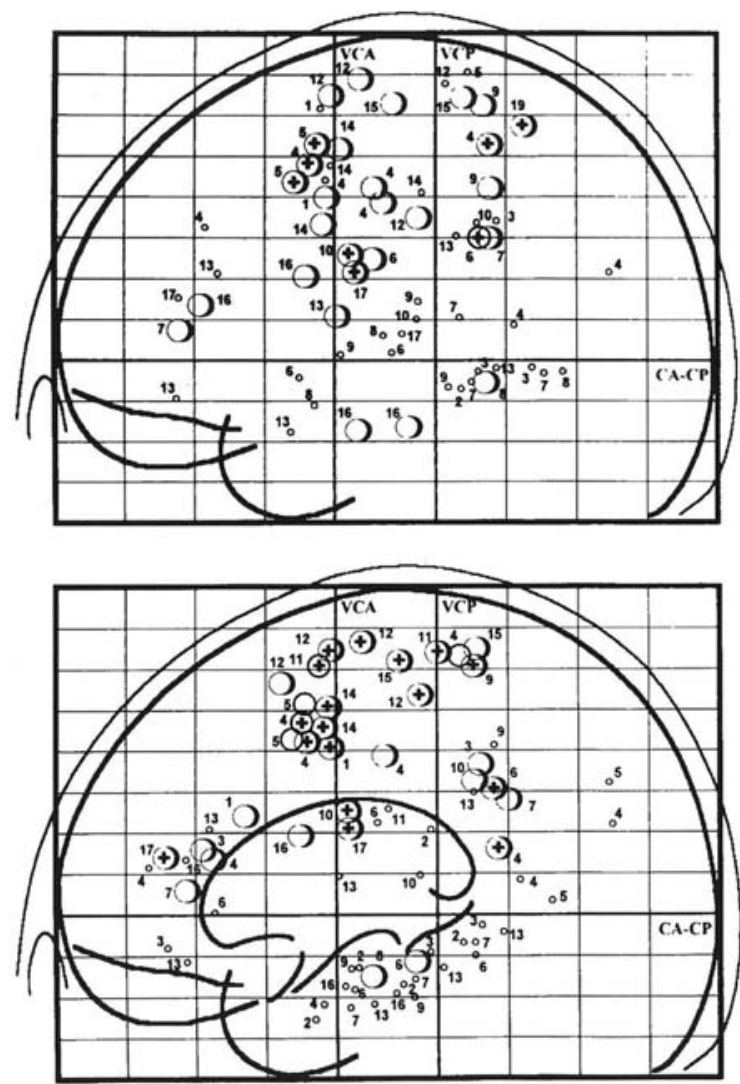

Figure 2. The cortical recording sites in the Bereitschaftspotential (BP) protocol in 26 patients. Upper part: Lateral view of the brain. Lower part: Sagittal aspect. In the supracallosal part, most of the internal contacts of multilead electrodes reached the mesial wall. In the lower part of the diagram, some of the recording points correspond to sites located more laterally. This schema presents the results in one or in both hemispheres. The numbers correspond to the individual patients. The large circles indicate the Movement Accompanying Potentials (MAP) occurrence. They are marked "+" when the BP preceded the MAP. The small circles indicate electrodes that recorded no slow potential. Reprinted from: Rektor, I. et al., (1998). Electroencephalography and Clinical Neurophysiology, 107, 277-286, with permission from Elsevier Science. tor et al., 1998; Rektor, 2003). MAP is also related to cognitive activity; its link to the execution of movement is indirect. In a series of direct intracerebral recordings with a repetitive simple movement, as well as with a repetitive complex movement, we localized the cerebral generators of the BP in a few cortical and subcortical regions. BP cortical generators were consistently displayed in the primary motor and sensory cortices contralateral to the writing hand, and in the bilateral supplementary motor area (SMA) and motor cingulate areas. BP occurred irregularly in the sensorimotor cortex ipsilateral to the writing hand, and in some premotor and prefrontal sites (for review see Rektor, 2003). Subcortical generators of BP were observed in the basal ganglia and in the posterior thalamus. MAP was always recorded after BP, but it also occurred independently in several pericentral regions, specifically in the premotor and anterior parietal cortices. There is an indirect link between the occurrence of cerebral generators of electrical activity and the hemodynamic changes measured by fMRI. The two phenomena (hemodynamic and electrophysiological changes) are different, but both occur when a given structure is active in a given task. We hypothesized that the fMRI changes should roughly overlap with the locations of the BP and MAP generators. These findings concur with the results of a recent combined event-related fMRI and intracerebral ERP study on P3 phenomena (Brázdil et al., 2005).

The comparison of our earlier electrophysiological data with this fMRI study might be hampered by the fact that the performed movements were not identical. Nevertheless, in our intracerebral recordings, various movements were used (hand flexion, foot flexion, complex hand movement with page turning) with BPs and MAPs that did not significantly differ, neither in the location in the brain, nor in the shape, duration, and amplitude (Rektor et al., 1994, 1998, 2001a; Rektor, 2003).

In this fMRI study, in connection with self-paced movement, we observed significant changes in the left pericentral region on the convexity (BA 3,1,2,4, and 40), as well as in the mesial wall (BA 6, 31), and nonsignificant changes at the right central convexity. This pattern of activation covers regions that are known to participate 


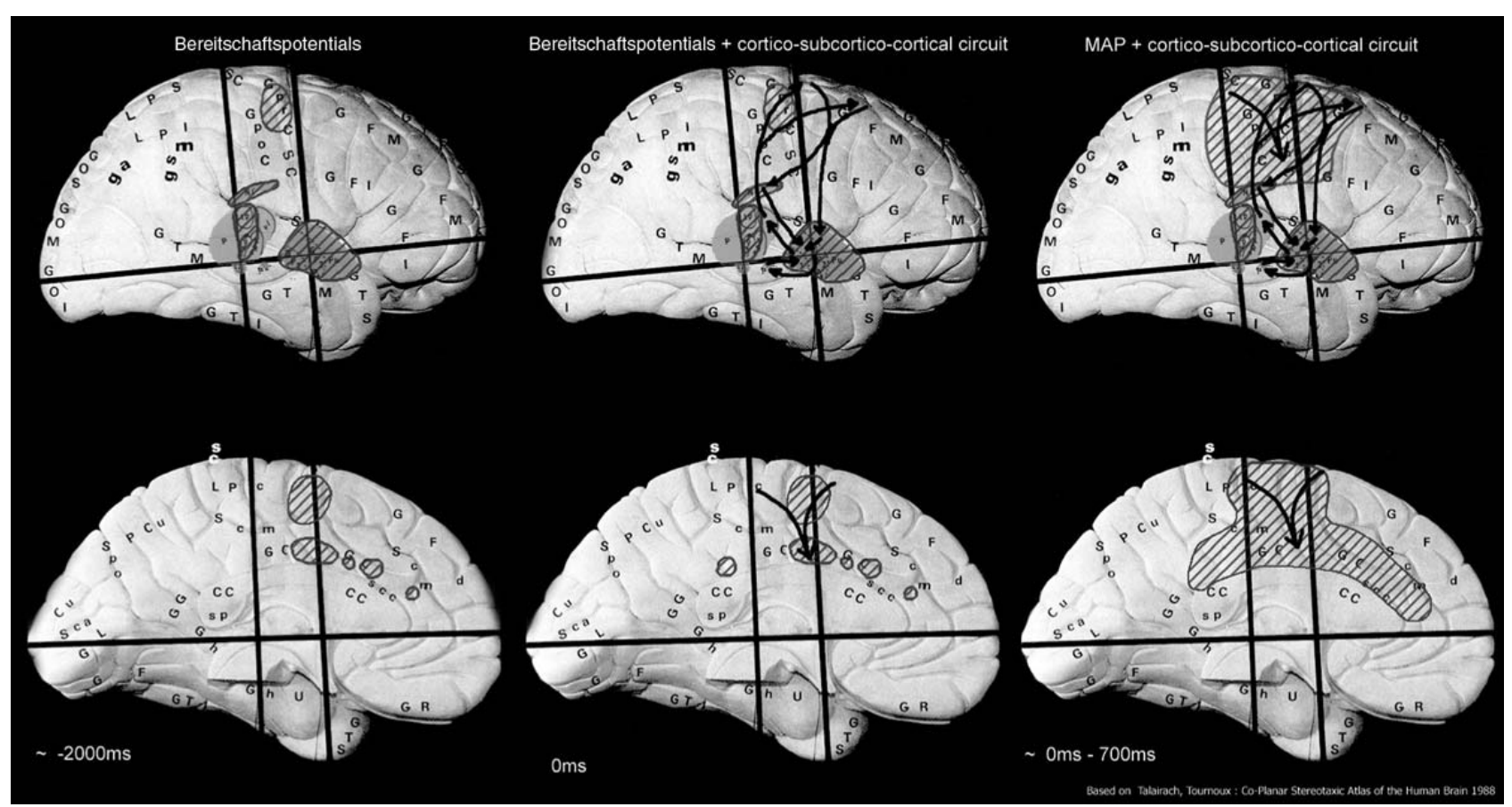

Figure 3. Schematic presentation of a long-lasting simultaneous activation of cortical and subcortical structures in the motor system before (BP) and during (MAP) self-paced movement of the contralateral hand. Left: Period starting approximately $2000 \mathrm{~ms}$ before the movement. Middle: The moment of the movement onset. In conjunction with the slow parallel activity, there is rapid sequential processing of information in the cortico-basal ganglia-thalamo-cortical circuitry. Right: The movement is executed. The rapid sequential processing continues while the slow activation spreads over more cortical areas. Striped: Slow parallel activity. Arrows: Rapid sequential activity. From: Rektor, I.(2003). Intracerebral recordings of the Bereitschaftspotential and related potentials in cortical and subcortical structures in human subjects. In M. Jahanshahi \& M. Hallett (Eds.), The Bereitschaftspotential (pp. 61-77). Kluwer Academic/Plenum, with permission.

in motor control: The primary sensorimotor cortex, the supramarginal gyrus, the SMA, and the underlying portion of the cingulate gyrus in the hemisphere contralateral to the movement, and to a lesser extent the sensorimotor region ipsilateral to the writing hand. This pattern corresponds to the electrical activity intracerebrally recorded in the BP protocol. The occurrence of MAP is slightly more widespread involving larger portions of prefrontal cortex (Figures 2 and 3). The nonsignificant hemodynamic changes in the sensorimotor cortex ipsilateral to the writing hand in our study may also result from the continuous tonic contraction of the left hand that was holding the writing pad while the right hand was writing. Moreover, the slight phasic changes of the muscle tone while holding the tablet are also possible. In a study by Foltys et al. (2003), a power grip was found to influence the ipsilateral sensorimotor activity. The unilateral occurrence of hemodynamic response in the SMA/cingulate may correspond to the asymmetrical amplitude of the BP, which is higher in the contralateral mesial central areas. In contrast with fMRI, the BP and MAP are irregularly recorded in some bilateral subtle prefrontal areas, including the anterior cingulate. It is not surprising that fMRI is less sensitive to hemodynamic changes that might underlie such irregular and subtle electrical activity chang- es. We were able to observe a small cluster of activity in the left inferior frontal gyrus (BA 44), but it did not reach statistical significance. The fMRI showed extensive activity in the bilateral supramarginal gyrus. The temporoparietal junction probably affects a remote control of other regions (Knight, 1997; Verleger et al., 1994). Other fMRI studies of self-initiated movements have shown similar cortical activation patterns, specifically the activity in the lateral and mesial pericentral cortices in the hemisphere contralateral to the movement; findings in other regions were more variable (Ball et al., 1999; Maccotta et al., 2001; Newton et al., 2002; Cunnington et al., 2002, 2003). In this study, we were not able to uncover any significant subcortical activation, while in an fMRI study with rapid sequential finger movements the hemodynamic changes were found in the putamen and midbrain (Cunnington et al., 2002), corresponding to the occurrence of BP and MAP generators in the basal ganglia (Rektor et al., 2001b). The movement used by Cunnington et al. differed from our protocol, and fMRI at 3T may be more sensitive. In any case, when comparing our fMRI study of a simple self-paced repetitive movement (writing dots) with intracerebral recordings of brain activity accompanying various self-paced movements, there is an evident overlap of results. 


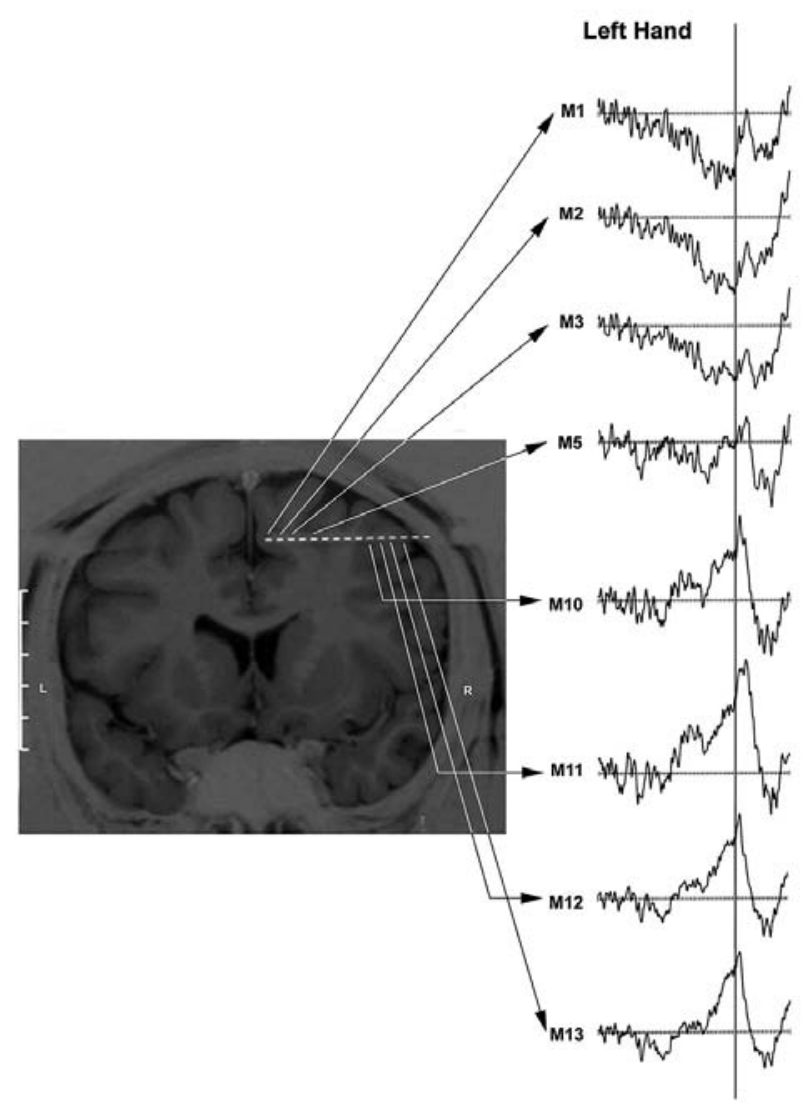

However the intracerebral recordings also displayed small BP generators in the frontal lobe (for details see Rektor, 2003), which did not concur with significant changes in the blood oxygenation level dependent (BOLD) signal. We observed a small bilateral BP in the dorsolateral prefrontal cortex (area 9, 46) from four electrodes in three patients; however, in four other recordings, no BP was recorded in these areas. While in many recordings, no BP generator could be identified in the lateral premotor cortex (Rektor et al., 1994, 1998, 2001a), in one case, we found $\mathrm{BP}$ in the bottom of a premotor sulcus that is usually inaccessible to such exploration (Figure 4). It is evident that in addition to the activation of large cortical regions, there are small generators distributed across various areas that cannot be discovered by fMRI. The BP/MAP and BOLD changes overlap in regions where the BP were displayed consistently, i.e., in nearly all recordings. In some other regions, the $\mathrm{BP}$ recordings were inconsistent, i.e., in the dorsolateral prefrontal cortex, where about half of the contacts displayed BP generators while the other half did not.

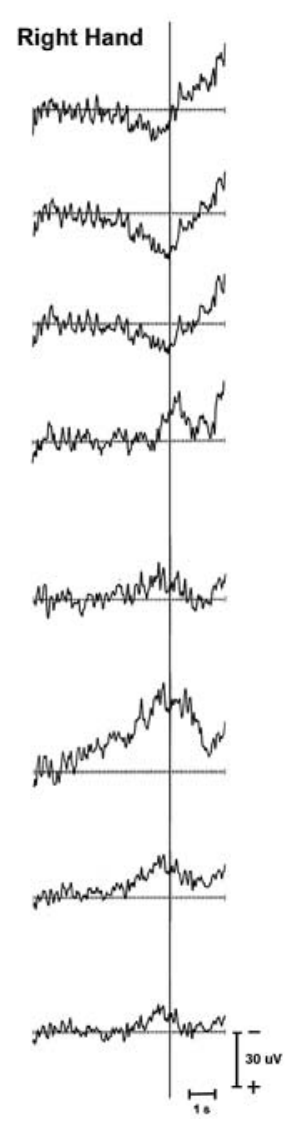

Figure 4. BP and MAP recorded on consecutive leads of an electrode passing from the SMA (contacts M1 and 2, and possibly M3), through the white matter (M4 to M9; only M5 is shown), through the premotor cortex in the bottom of a vertically oriented sulcus (M11) into the premotor cortex of the convexity (M13). Left: MR imaging of the brain with a superimposed reconstruction of the electrode position based on MR artifacts caused by the electrode (not shown). Middle: Recording triggered by the movement of the contralateral (left) hand. Right: Recording triggered by the movement of the ipsilateral (right) hand. Note the BP with maximal amplitude (a generator) in the SMA (M2) and in a part of the premotor cortex (M11), although no generator was recorded in the premotor cortex of the convexity. No BP, or only small far-field potentials, were recorded in the white matter (M5; other contacts not shown). The amplitude of all BP is higher with contralateral than with ipsilateral hand movement.

From: Rektor, I. (2003). Intracerebral recordings of the Bereitschaftspotential and related potentials in cortical and subcortical structures in human subjects. In M. Jahanshahi and M. Hallett (Eds.), The Bereitschaftspotential (pp. 61-77). Kluwer Academic/Plenum, with permission.

In these regions the BOLD changes were not significant. This does not mean that there were no hemodynamic changes in the studied region at all, but that if present they were insufficient for reaching the threshold for statistical significance. This is not surprising when bearing in mind that the $\mathrm{BP}$ was present in a part of sites in a given region.

It is not surprising that electrophysiological studies are more sensitive in uncovering small active areas, while the fMRI that indirectly measures activity of larger populations of neurons has better spatial resolution. The spatial limitations of the intracerebral electrodes are the result of the fact that the electrodes are submerged in the cerebral tissue and record in their immediate vicinity. Electrophysiological techniques with intracranial recordings may reveal even very subtle generators of electrical activity in sites where fMRI results are negative, because the concurrent hemodynamic changes do not reach statistical significance. The MRI may reliably reveal areas that are active during a task but a negative result does not exclude the active involvement of other regions. 


\section{Acknowledgments}

The study was supported by Research Program MSM0021622404.

\section{References}

Ball, T., Schreiber, A., Feige, B., Wagner, M., Lücking, H.C., \& Kriteva-Feige, R. (1999). The role of higher-order motor areas in voluntary movement as revealed by high-resolution EEG and fMRI. NeuroImage, 10, 682-694.

Brázdil, M., Dobšík, M., Mikl, M., Daniel, P., Pažourková, M., Krupa, P., \& Rektor, I. (2005). Depth ERPs and fMRI comparative study of auditory oddball task. NeuroImage, 26, 285293.

Cunnington, R., Windischberger, C., Deecke, L., \& Moser, E. (2004). The preparation and execution of self-initiated and externally-triggered movement: A study of event-related fMRI. NeuroImage, 15, 373-385.

Cunnington, R., Windischberger, C., Deecke, L., \& Moser, E. (2003). The preparation and readiness for voluntary movement: A high-field event-related fMRI study of the Bereitschafts-BOLD response. NeuroImage, 20, 404-412.

Foltys, H., Meister, I., Weidemann, J., Sparing, R., Thron, A., Wilmes, K., Töpper, R., Hallett, M., \& Boroojerdi, B. (2003). Power grip disinhibits the ipsilateral sensorimotor cortex: A TMS and fMRI study. Neurolmage, 19, 332-340.

Knight, R.T. (1997). Distributed cortical network for visual attention. Journal of Cognitive Neuroscience, 9(1), 75-91.

Kornhuber, H.H., \& Deecke, L. (1965). Hirnpotentialänderungen bei Willkürbewegungen und passiven Bewegungen den Menschen: Bereitschaftspotential und reafferente Potentiale [Brain potentials in voluntary and passive movements in human subjects: Bereitschaftspotential and reafferent potential]. Pflügers Archiv, 284, 1-17.

Libet, B. (1985). Unconscious cerebral initiative and the role of conscious will in voluntary action. Behavioral and Brain Sciences, 8, 529-566.

Maccotta, L., Zacks, J., \& Buckner, R. (2001). Rapid self-paced event-related functional MRI: Feasibility and implications of stimulus- versus response-locked timing. NeuroImage, 14, $1105-1121$.
Newton, J., Sunderland, A., Butterworth, S., Peters, A., Peck, K., \& Gowland, P.(2002). A pilot study of event-related functional magnetic resonance imaging of monitored wrist movements in patients with partial recovery. Stroke, 33, 2881-2887.

Rektor, I., Feve, A., Buser, P., Bathien, N., \& Lamarche, M. (1994). Intracerebral recording of movement related readiness potentials: An exploration in epileptic patients. Electroencephalography and Clinical Neurophysiology, 90, 273-283.

Rektor, I., Louvel, J., \& Lamarche, M. (1998). Intracerebral recording of potentials accompanying simple limb movements: A SEEG study in epileptic patients. Electroencephalography and Clinical Neurophysiology, 107, 227-286.

Rektor, I., Bareš, M., Kanovský, P., \& Kukleta, M. (2001a). Intracerebral recording of readiness potential induced by a complex motor task. Movement Disorders, 16, 698-704.

Rektor, I., Kaňovský, P., Bareš, M., Louvel, J., \& Lamarche, M. (2001b). Evoked potentials, ERP, CNV, readiness potential, and movement accompanying potential recorded from the posterior thalamus in human subjects. A SEEG study. Neurophysiologie Clinique, 31, 1-9.

Rektor, I., Bareš, M., \& Kubová, D. (2001c). Movement-related potentials in the basal ganglia: A SEEG readiness potential study. Clinical Neurophysiology, 112, 2146-2153.

Rektor, I. (2003). Intracerebral recordings of the Bereitschaftspotential and related potentials in cortical and subcortical structures in human subjects. In M. Jahanshahi and M. Hallett (Eds.) The Bereitschaftspotential (p. 61-77). New York: Kluwer Academic/Plenum

Verleger, R., Heide, W., Butt, C., \& Kömpf, D. (1994). Reduction of $\mathrm{P} 3_{\mathrm{b}}$ in patients with temporo-parietal lesions. Cognitive Brain Research, 2, 103-116.

Accepted for publication: November 11, 2005

Ivan Rektor

First Department of Neurology, Masaryk University St. Anne's Hospital, Pekarska 53

65691 Brno

Czech Republic

Tel. +420 543182623

Fax +420543182624

E-mail irektor@med.muni.cz 